\title{
The circulating metabolome of human starvation
}

\author{
Matthew L. Steinhauser, ${ }^{1,2,3,4}$ Benjamin A. Olenchock, ${ }^{2,3,4}$ John O'Keefe, ${ }^{1}$ Mingyue Lun, ${ }^{1}$ \\ Kerry A. Pierce, ${ }^{4}$ Hang Lee, ${ }^{3,5}$ Lorena Pantano, ${ }^{6}$ Anne Klibanski, ${ }^{3,7}$ Gerald I. Shulman, ${ }^{8}$ Clary B. Clish,${ }^{4}$ \\ and Pouneh K. Fazeli ${ }^{3,7}$ \\ 'Department of Medicine, Division of Genetics, and 'Department of Medicine, Division of Cardiovascular Medicine, Brigham \\ and Women's Hospital, Boston, Massachusetts, USA. ${ }^{3}$ Harvard Medical School, Boston, Massachusetts, USA. ${ }^{4}$ Broad \\ Institute of MIT and Harvard, Cambridge, Massachusetts, USA. ${ }^{5}$ MGH Biostatistics Center, Boston, Massachusetts, USA. \\ ${ }^{6}$ Department of Biostatistics, Harvard T.H. Chan School of Public Health, Boston, Massachusetts, USA. ${ }^{7}$ Neuroendocrine \\ Unit, Department of Medicine, Massachusetts General Hospital, Boston, Massachusetts, USA. ${ }^{8}$ Departments of Internal \\ Medicine and Cellular and Molecular Physiology and Howard Hughes Medical Institute, Yale University School of Medicine, \\ New Haven, Connecticut, USA.
}

The human adaptive starvation response allows for survival during long-term caloric deprivation. Whether the physiology of starvation is adaptive or maladaptive is context dependent: activation of pathways by caloric restriction may promote longevity, yet in the context of caloric excess, the same pathways may contribute to obesity. Here, we performed plasma metabolite profiling of longitudinally collected samples during a 10-day, 0-calorie fast in humans. We identify classical milestones in adaptive starvation, including the early consumption of gluconeogenic amino acids and the subsequent surge in plasma nonesterified fatty acids that marks the shift from carbohydrate to lipid metabolism, and demonstrate findings, including (a) the preferential release of unsaturated fatty acids and an associated shift in plasma lipid species with high degrees of unsaturation and (b) evidence that acute, starvation-mediated hypoleptinemia may be a driver of the transition from glucose to lipid metabolism in humans.

Conflict of interest: The authors have declared that no conflict of interest exists.

Submitted: April 3, 2018 Accepted: July 10, 2018 Published: August 23, 2018

Reference information: JCI Insight. 2018;3(16):e121434. https://doi.org/10.1172/jici. insight.121434.

\section{Introduction}

Our evolutionary past was marked by periods of famine. The evolution of a coordinated set of responses to periods of starvation was critical to our survival. However, the physiology of starvation may be of relevance beyond epidemic famines, which remain a significant threat to public health (1). Indeed, some aspects of the starvation response may account for the paradoxical beneficial effects gained from caloric restriction, including longevity, which appear to be conserved across diverse species (2). Whether the remarkable ability of humans to survive extended periods of starvation is adaptive or maladaptive, however, may be context dependent. The more common scenario in the developed world is now a state of nutrient excess and obesity, which may be exacerbated by the same responses that enable survival during starvation. Therefore, understanding the physiology of starvation may reveal mechanisms underlying the obesity epidemic and reveal pathways involved in longevity.

Human starvation is marked by an early period of glycogenolysis and gluconeogenesis (3, 4). By 2-3 days into a fast, fatty acids released from lipid stores become the primary source of fuel; this critical transition to lipid metabolism allows for protein sparing during prolonged periods of starvation (3). What are the hormonal signals that mediate this transition from glucose to lipid metabolism? Whereas hypoinsulinemia, glucagon, and glucocorticoids are thought to be key mediators of gluconeogenesis during starvation $(3,5-8)$, less is known about the transition from gluconeogenesis to ketogenesis. Recent data in a rodent model suggest that hypoleptinemia, which in turn drives hypothalamic-pituitary-adrenal (HPA) axis activation, may be a necessary mediator of adipose tissue lipolysis (9), an important step in the transition to lipid metabolism during starvation. However, the practical challenges surrounding studies of human starvation, coupled with key differences between model organisms and humans, account for the ongoing uncertainty surrounding the regulatory mechanisms that govern the metabolism of starvation in humans. 
The circulating metabolome, which reflects systemic metabolic processes, represents one aspect of starvation physiology that is incompletely understood. Prior studies have demonstrated the effects of prolonged fasting on small groups of metabolites (10). This provided rationale to revisit the human starvation response using large-scale metabolite profiling together with clinical phenotyping to characterize human starvation with a previously unprecedented level of granularity. We studied individuals undergoing a 0 -calorie, 10-day fast and present longitudinal metabolite data. We examined classes of metabolites in turn including fatty acids and fatty acid catabolites, lipids, and amino acids - and demonstrate preferential positive shifts in unsaturated fatty acid and lipid species during fasting. We then categorized metabolites based on their temporal dynamics, independent of metabolite class, elucidating discrete clusters with specific hormonal correlates. This unbiased assessment revealed that acute, starvation-mediated hypoleptinemia may be a driver of the transition from glucose to lipid metabolism in humans.

\section{Results}

Starvation results in global shifts in circulating metabolites. We collected serial plasma samples from healthy human volunteers during a 10-day inpatient fast and performed metabolite profiling, inclusive of polar and nonpolar species (Figure 1A). The current analysis includes 12 subjects, 8 of whom completed the entire 10 -day fasting protocol. Four subjects stopped early due to self-removal from the study $(n=2)$, study staff judgment due to degree of weight loss ( $n=1$, completed 7 days of the fast), and transaminase elevation at baseline $(n=1)$. Baseline clinical characteristics, previously reported for a subset of this study population (11), are provided in Supplemental Table 1 (supplemental material available online with this article; https://doi.org/10.1172/jci.insight.121434DS1). Samples were collected during a study visit 10 days prior to fasting initiation (prebaseline visit); upon admission for the fasting visit (baseline/day 0); in the mornings of fasting days 1, 2, 3, 5, 7, 9, and 10 (final fast day); and then again in the morning following refeeding (Figure 1, A and B). Subjects lost between $7.0 \%$ and $11.7 \%$ of their initial body weight. Over the course of the study, 351 metabolites significantly deviated from baseline measurements $(P<0.05$, adjusted for multiple testing) (Figure 1C), and 292 metabolites met a narrower criteria, consisting of an adjusted $P$ value of less than 0.05 and a $\log$ fold change of $>0.5$ (Figure 1, C and D). Metabolite changes became particularly evident at day 2 of fasting and beyond (Figure 1, B and C). Diverse classes of lipid metabolites were represented, including triacylglycerols (TAG) and diacylglycerols, fatty acids, acyl carnitines, cholesterol esters (CE), and phospholipids (PL). Additional represented classes included those related to amino acid, nucleic acid, and bile acid metabolism.

We next performed a principal component analysis (PCA), examining metabolite profiles as a function of time, using two lead principal components (Figure 1E). Over the course of the fast, the subjects converged into a more discrete cluster (Figure 1E, blue dots). We then tested correlations between measured clinical covariates and the lead principal components. Factors indicative of the duration/scale of starvation, including fasting duration and degree of weight loss, correlated with the lead principal component (Supplemental Figure 1). The strongest biochemical and/or hormonal correlates of the principal components were circulating ketones and nonesterified fatty acids (NEFA). Collectively, these data are consistent with starvation-mediated convergence toward common adaptive mechanisms involving ketones and NEFA.

Starvation-induced mobilization and catabolism of fatty acids. Prior studies have demonstrated global changes in serum lipids and fatty acids with human starvation (12), including elevations in NEFA, which we previously confirmed in the current study population (11). In this study, we provide analyses of individual lipids and lipid metabolites. We observed a fasting-mediated increase in individual fatty acids that was nearly uniform by fasting day 2 and persisted for the duration of the fast (Figure 2A). We hypothesized that the lipolytic release of fatty acids from adipose tissue triglyceride stores would be agnostic to fatty acid identity, in which case changes in circulating levels of individual fatty acids relative to their baseline values would be similar across fatty acid species. Instead, we observed more profound augmentation of circulating fatty acids as a function of an increasing number of double bonds (Figure 2, B and C). Consistent with progressive fatty acid oxidation, we observed a sustained surge in circulating acyl carnitines of various lengths and degrees of saturation with fasting (Figure 2D). This surge included short-chained acyl carnitines and end products, such as $\mathrm{C} 4: 0(\mathrm{OH})$, a ketone body that correlates with more commonly measured ketone species, such as $\beta$-hydroxybutyrate (Figure 2E) (13). The increase in fully saturated acyl carnitines was also attenuated relative to unsaturated species of similar length (Supplemental Figure 2), although the effect of double bond content was not as strong as that observed for fatty acids. Because the preferential surge in unsaturat- 
A

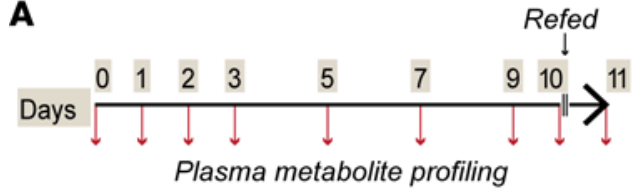

B Fasting Day

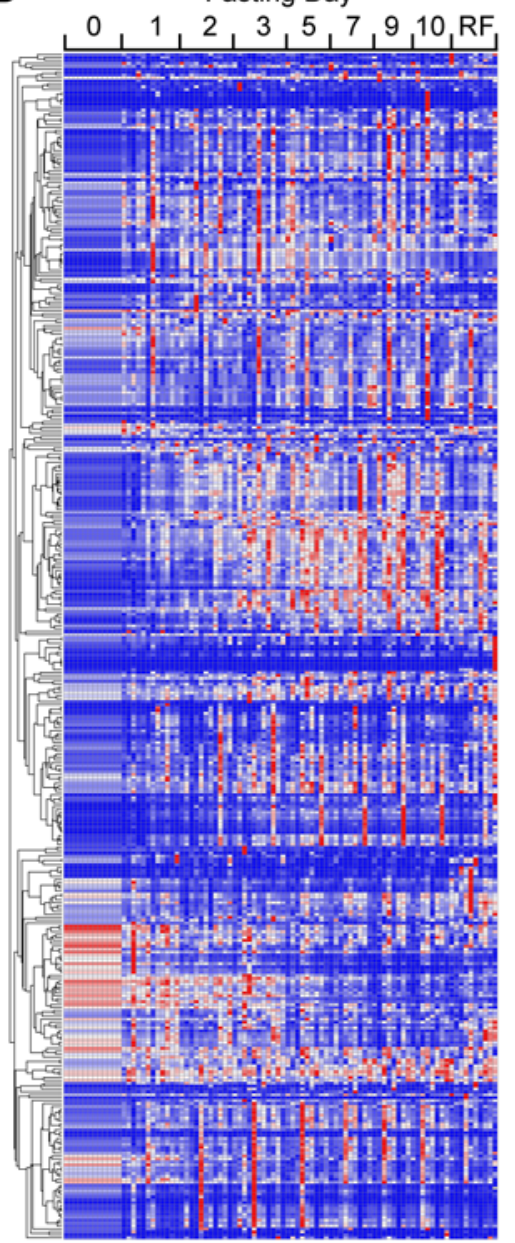

row min

D

E
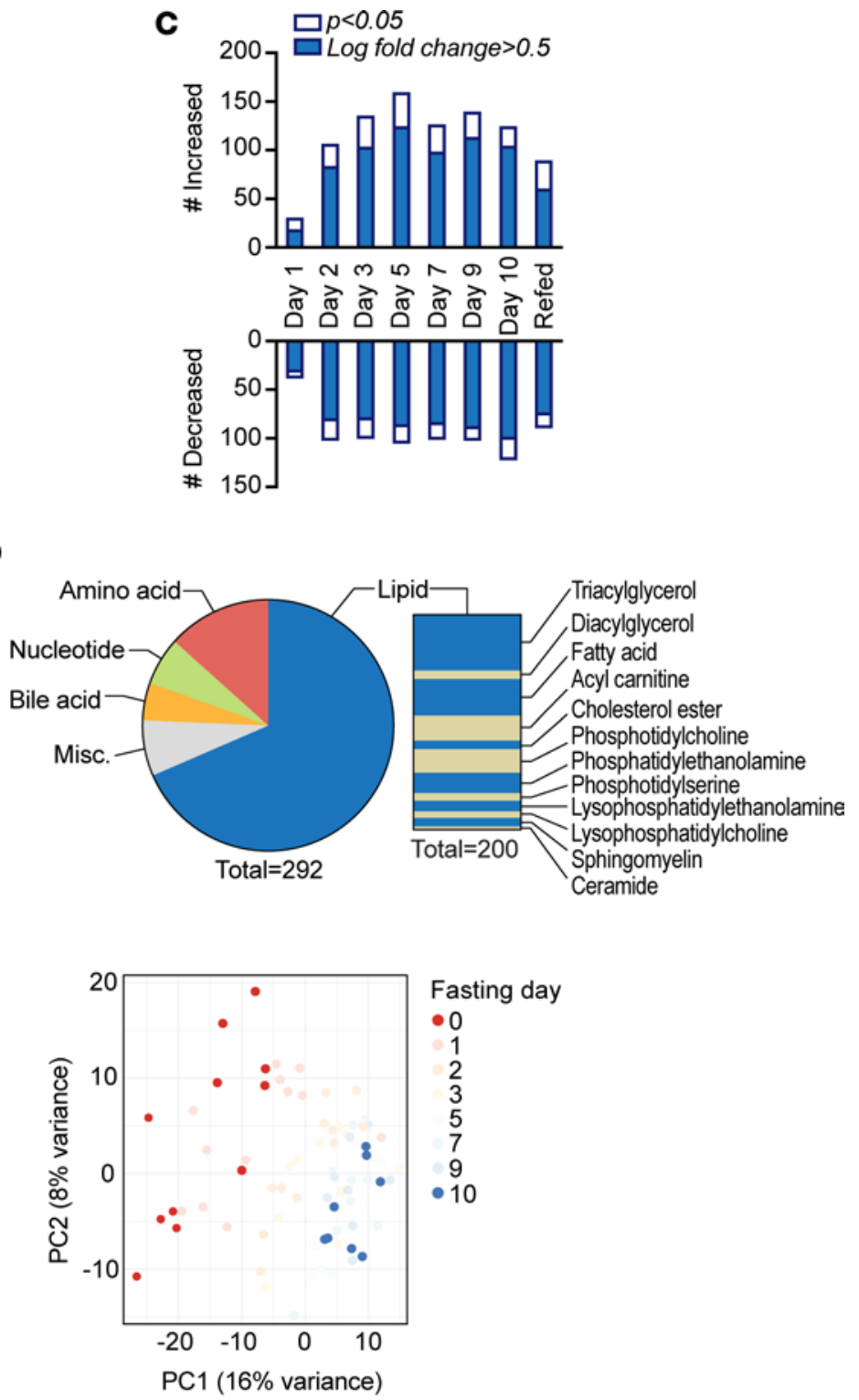

Figure 1. Starvation results in global shifts in circulating metabolites. (A) Schematic time line of the human-fasting protocol (day 0 , baseline day; day 10 , final fast day). (B) Heatmap displaying plasma metabolites with fasting $(n=544)$. RF, refed day 1. (C) The number of metabolites deviating from baseline (day 0 ) at each time point (adjusted $P<0.05$ ). Blue bars, subset that also deviated by log fold change $>0.5$. (D) Pie chart showing class breakdown of metabolites represented by blue bars in C. (E) Principal component analysis (PCA) plot of leading principal components (PC1 and PC2). Dark red dots, baseline; dark blue dots, day 10 of fast; lighter shades, intermediate time points. With fasting, subjects converged to the lower right quadrant (dark blue dots).

ed fatty acids was coupled with a directionally consistent pattern of rise in their catabolic byproducts, these data are consistent with preferential release and catabolic flux of unsaturated fatty acids from lipid stores.

In addition to providing fuel, long-chain polyunsaturated fatty acids (PUFA), such as arachidonic acid, provide substrate for enzymatic reactions that produce eicosanoid signaling molecules. Similar to other fatty acid species, circulating long-chain PUFA, including arachidonic acid (C20:4), increased with fasting (Figure 2F). Coincident with this surge, we also observed increased levels of eicosanoids (Figure 2G). Whether the augmentation of eicosanoids is a nonspecific spillover effect of increased substrate availability or due to a coordinated fasting-mediated stimulation of the biosynthetic pathway is not revealed by these data.

Starvation-mediated shift to TAG and CE lipids with high double bond content. Prior studies have demonstrated modest changes in plasma lipids, including TAG, with fasting (14). We previously measured TAG in this cohort of subjects at baseline and at the end of the fast using a clinical laboratory assay and found a trend 

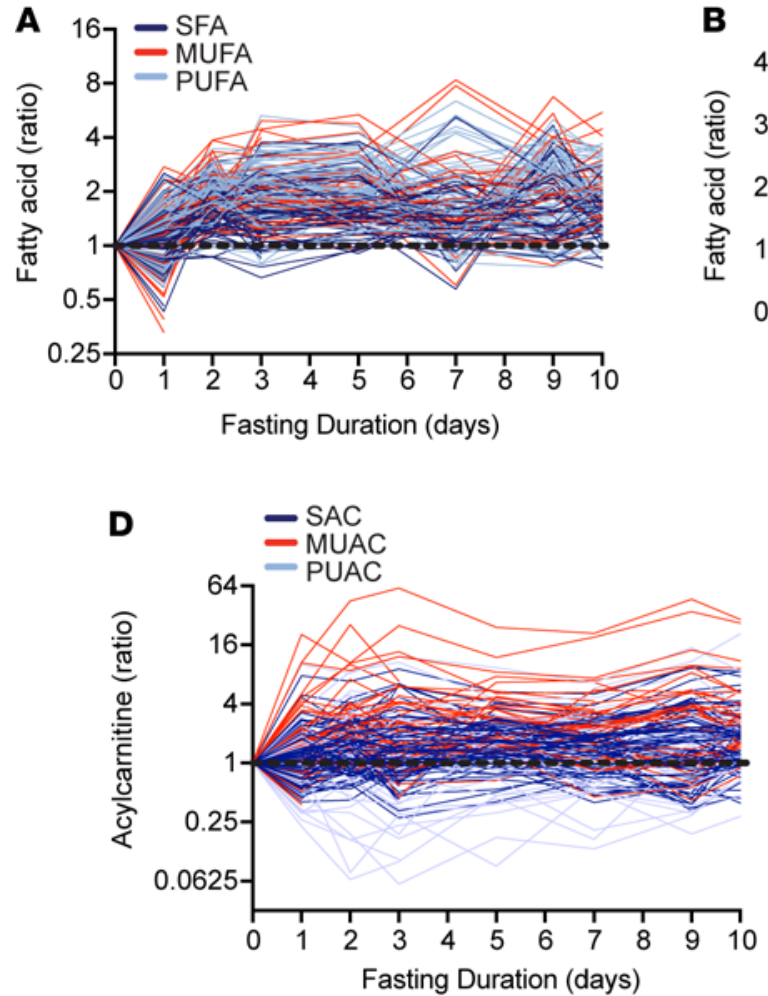

$\mathbf{F}$

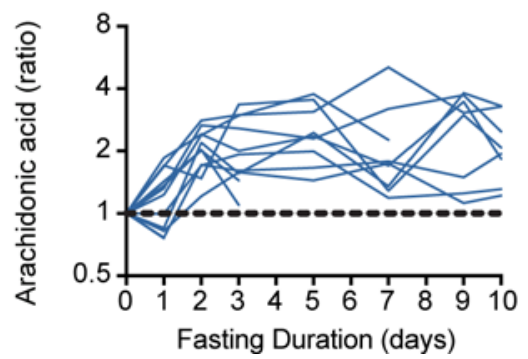

B

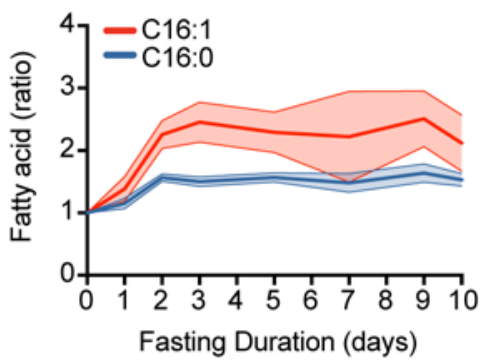

C

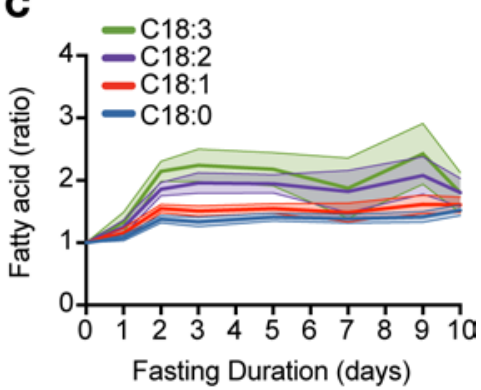

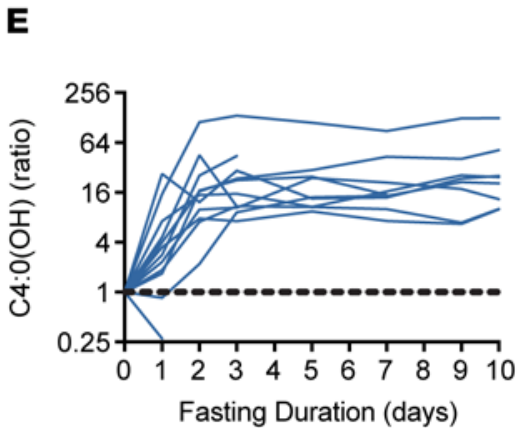

G

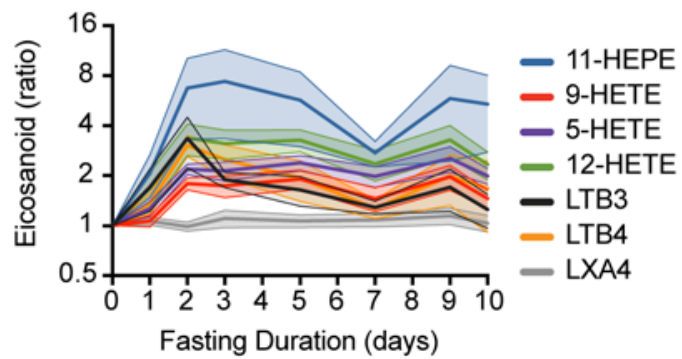

Figure 2. Starvation-induced mobilization and catabolism of fatty acids. (A) Spaghetti plot of fatty acids relative to baseline (dashed line) for each subject, grouped by degree of saturation: saturated (SFA), monounsaturated (MUFA), and polyunsaturated (PUFA). (B) Mean change from baseline ( \pm SEM) of fatty acids comprised of 16 carbons (C16). The monounsaturated C16:1 increases more than fully saturated palmitic acid (C16:0). The number after the colon denotes the double bond number. (C) Mean relative change from baseline $( \pm$ SEM) of fatty acids comprised of 18 carbons (C18), showing increase in fatty acid levels as a function of the double bond number. (D) Spaghetti plot of acyl carnitines relative to baseline (dashed line) for each subject, grouped by degree of saturation: saturated, monounsaturated, and polyunsaturated. (E) Spaghetti plot of $[4: 0(\mathrm{OH})$ relative to baseline (dashed line) for each subject. (F) Spaghetti plot of arachidonic acid relative to baseline (dashed line) for each subject. (G) Mean change from baseline ( \pm SEM) for plasma eicosanoids.

toward increased TAG (11). The standard clinical laboratory assay for TAG involves measurement of glycerol after acyl chain hydrolysis as a proxy for total TAG. In contrast, the lipidomics platform utilized here enables identification of intact lipid species, inclusive of TAG, CE, and a range of PL classes, with each individual species defined by carbon content and carbon-carbon double bond number (degree of unsaturation) (15). In contrast to fatty acids, lipid dynamics were often directionally discordant within classes (Figures 3 and 4), including plasma TAG and CE (Figure 3). The species were approximately evenly split with respect to direction of change with fasting; however, we observed a striking pattern dependent on the number of double bonds and carbon content. TAG with a higher number of double bonds (unsaturated) tended to increase with fasting, whereas more saturated TAG tended to decrease with fasting. At the extremes, TAG C60:12 increased by approximately 30-fold, whereas fully saturated TAG C44:0 declined by approximately 2-fold. Although there were fewer discrete and detectable $\mathrm{CE}$ analytes, a similar pattern of dependence on double bond content emerged, with more pronounced increases in CE containing 20-22 carbons and $\geq 4$ double bonds. 
PL are a third major class of lipids. Critical to membrane structure and function, PL or their derivatives may also serve signaling functions, and their fatty acyl components represent an additional potential energy source. We observed divergent effects of starvation on plasma levels of PL, both between and within PL classes (Figure 4). Many phosphatidylcholine (PC) and phosphatidylethanolamine (PE) plasmalogens decreased during fasting, including several by greater than 2-fold (PC 34:5, PE 34:2, PE 34:3, PE 36:3, PE 36:4); although, some plasmalogens increased, albeit to less than double baseline (Figure 4, A and G). Fasting also led to a reduction in many of the $\mathrm{PC}$ and $\mathrm{PE}$, without a corresponding increase in the respective byproducts of catabolic fatty acid release, the lysophosphatidylcholines and lysophosphatidylethanolamines (Figure 4, B and C). Despite a decline in many PC and PE, a subset with high-carbon content and high degree of unsaturation ( $>4$ double bonds) demonstrated a modest increase with fasting, similar to that observed for TAG and CE (Figure 4B). In contrast, the sphingomyelins (SM), another lipid class, demonstrated a consistent increase in plasma levels during fasting (Figure 4, A and F), which was not related to carbon content or saturation.

Collectively, the lipidomic data presented here provide a comprehensive view of the fasting-mediated effects on circulating lipids. The most consistent theme that emerged was the fasting-mediated shift in favor of plasma lipids of high-carbon and high double bond content, a pattern observed for TAG, CE, PC, and $\mathrm{PE}$. While most of the metabolite shifts that transpired with fasting converged toward baseline values with refeeding, the shifts in favor of unsaturated, high-carbon content CE and TAG persisted after refeeding (Figure 3C and Supplemental Table 2).

An amino acid surge as a candidate driver of the shift to fatty acid and ketone utilization. The shift to ketone production by the liver represents a critical response that enables humans to survive prolonged periods of starvation. This provided rationale to search for candidate metabolite participants in the shift to fatty acid utilization and ketogenesis. We first explored this by assessing for individual metabolite correlates to previously measured serum NEFA and ketones ( $\beta$-hydroxybutyrate) (11). We found significant positive correlations (adjusted $P<0.05)$ with NEFA $(n=62)$ and ketones $(n=57)$ (Supplemental Table 3). A subset of the metabolites that correlated with NEFA were themselves fatty acids $(n=25$ of 62$)$ and, therefore, components of the global NEFA measurement or downstream fatty acid metabolites $(n=16$ of 62$)$, such as acyl-carnitines. Consistent with the fact that ketones are a product of fatty acid catabolism, many of the ketone correlates were also intermediates in fatty acid catabolism $(n=19$ of 57). Similarly, 7 of the ketone metabolite correlates were either ketogenic amino acids (e.g., leucine) or related metabolites (e.g., aminocaproic acid, lysine metabolite). Many metabolites $(n=25)$ were common to both lists. Though much of this overlap is explainable by the substrate-product relationship between NEFA and ketones, several amino acids did not fit this categorization.

Prior studies have demonstrated amino acid release from tissues, including skeletal muscle, during the first few days of human starvation (16). Unlike the catabolic release of fatty acids from lipids, which results in a directionally uniform surge in plasma levels (Figure 2), amino acid measurements revealed variable patterns of amino acid dynamics (Figure 5), many of which have been previously documented by classic studies of prolonged starvation conducted in obese individuals (10) (Supplemental Table 4). Whereas leucine, isoleucine, valine, asparagine, glycine, methionine, phenylalanine, tyrosine, and lysine increased with fasting, other amino acids, including alanine, glutamate, glutamine, and tryptophan decreased. Even for directionally concordant amino acids, there were differences in timing of change. For example, branched-chain amino acids (BCAA) increased within 1-2 days, whereas tyrosine, glycine, asparagine, methionine, and lysine surged at approximately day 5.

Even when categorized by their potential utilization as substrates for gluconeogenesis, ketogenesis, or both (Figure 5A), we observed nonuniformity in amino acid dynamics, suggesting a role beyond fuel utilization alone. Tryptophan represents one such example; plasma tryptophan levels decreased by fasting day 2 and then plateaued (Figure 5A), coincident with increases in some downstream tryptophan metabolites, including members of the kynurenine pathway (Supplemental Figure 3). Although we did not see a significant increase in serotonin, a tryptophan metabolite and signaling molecule, there was a trend toward an increase on fasting day 3 (adjusted $P=0.07$ ). The fact that an essential amino acid, such as tryptophan, may be preferentially shunted down these pathways in the absence of dietary tryptophan intake raises the question of whether one or more of the associated metabolites plays an important regulatory role in fasting (17-19).

BCAA demonstrated a uniform directional and temporal change, with a surge within 1-2 days of fasting onset (Figure 5A). Importantly, we observed a coincident increase in several downstream BCAA catabolites, including $\beta$-aminoisobutyric acid (BAIBA) (Figure 5B and Supplemental Figure 4), suggesting that the BCAA surge likely arises from increased release into circulation rather than a block in downstream catabolism. Given prior evidence suggesting a role for BAIBA in fatty acid oxidation and ketone production $(20,21)$, we 
A

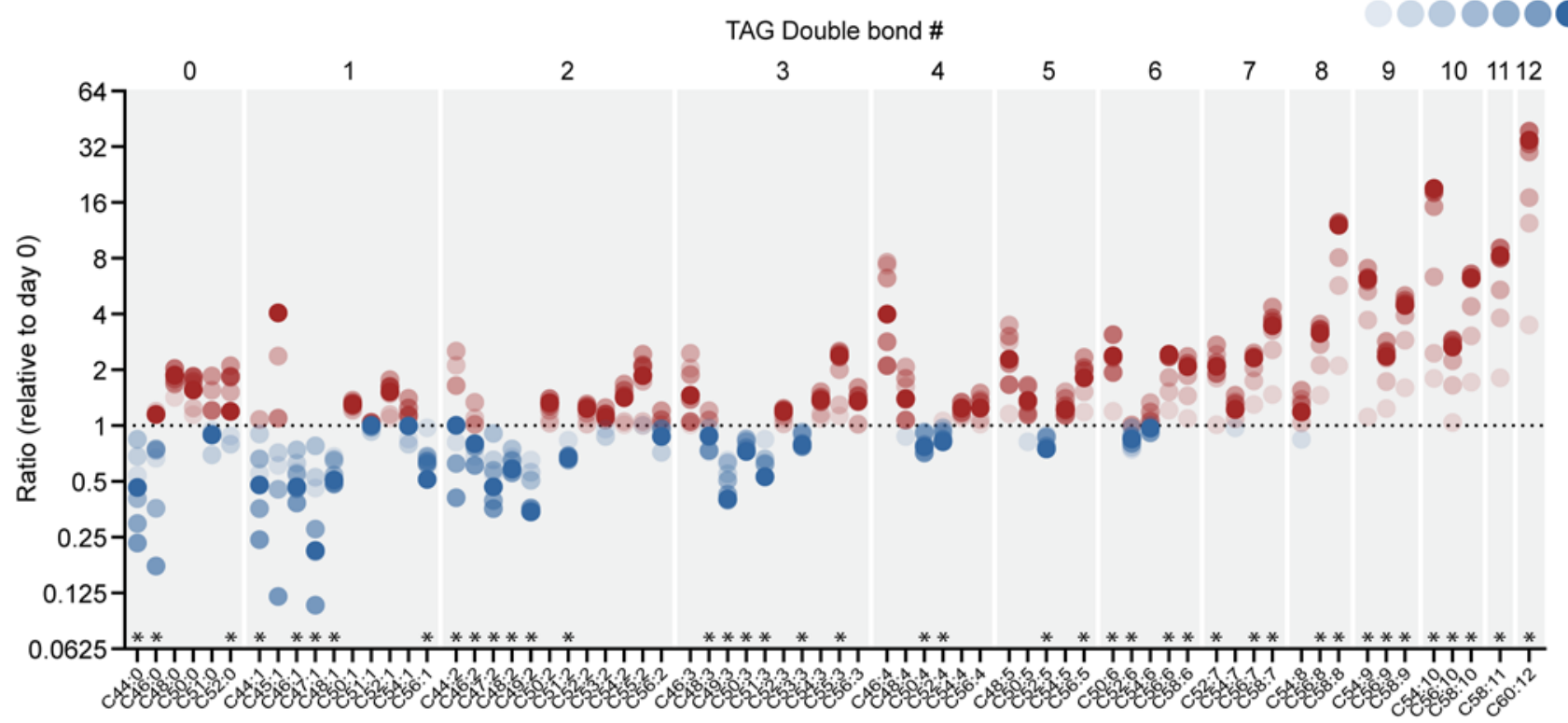

B

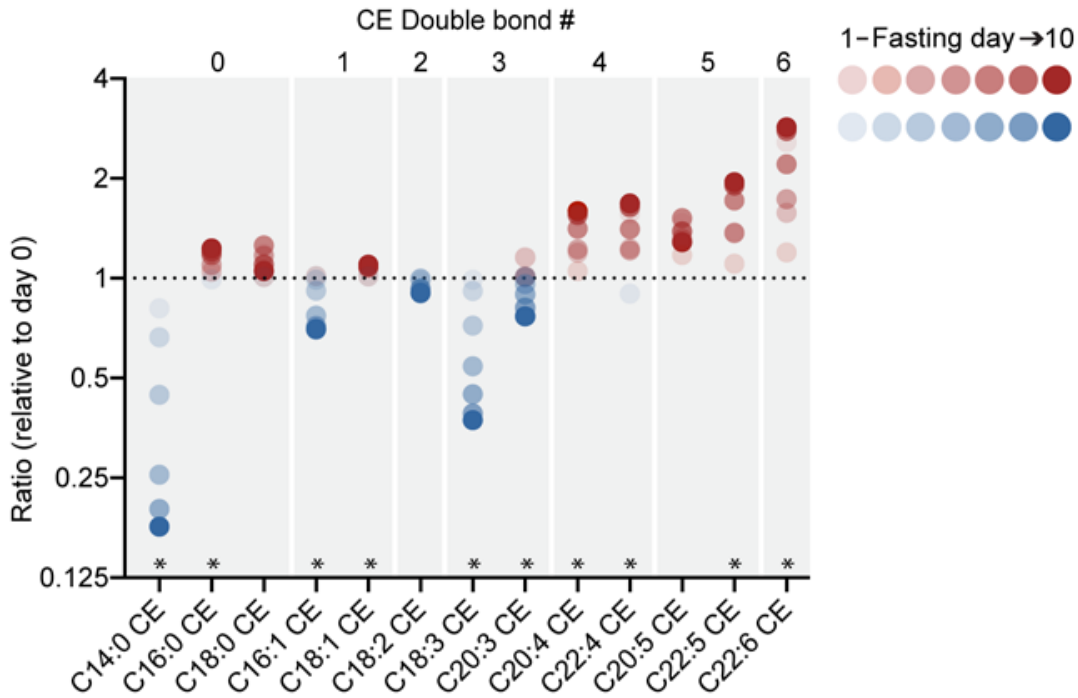

C
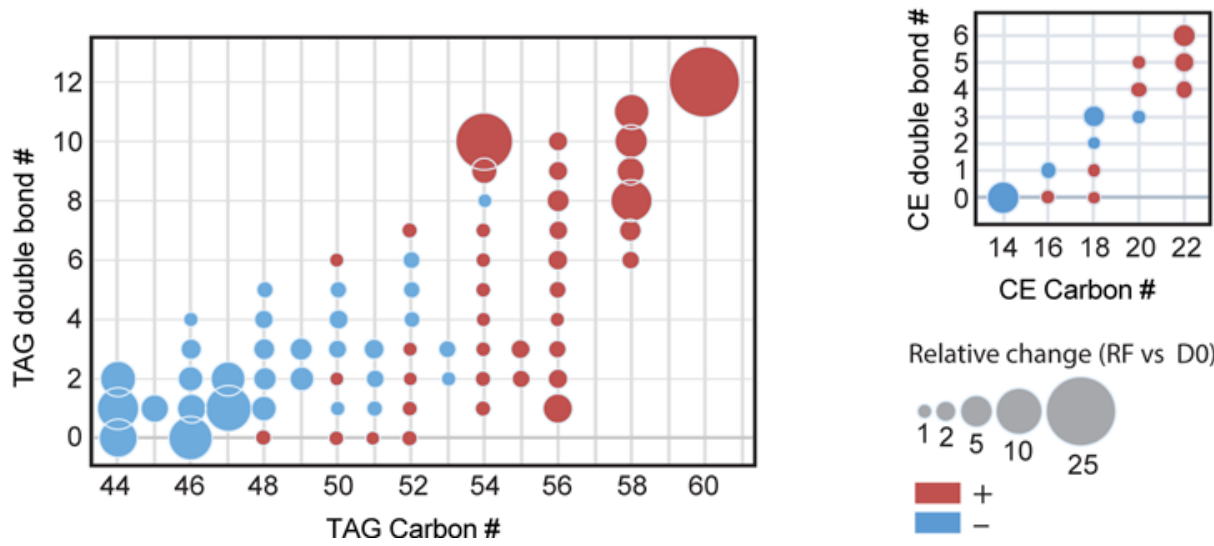

Relative change (RF vs Do)

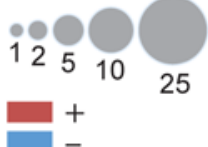


Figure 3. Starvation-mediated shift to triacylglycerol and cholesterol ester lipids with high double bond content. (A) Each dot corresponds to mean triacylglycerol (TAG) levels relative to baseline (dashed line). (B) Each dot corresponds to mean cholesterol ester (CE) levels relative to baseline (dashed line). (A and B) Blue dots signify negative levels relative to baseline; red dots signify positive levels relative to baseline. Dots become progressively darker as a function of fasting duration. Lipids are grouped by number of double bonds (shown at the top). Within each double bond group, lipids are ordered by ascending number of carbons. ${ }^{*} P<0.05$, comparing day 10 to baseline (false discovery rate adjustment for multiple comparisons). (C) Bubble graphs displaying mean TAG levels and mean CE levels after refeeding (RF), relative to baseline (DO). Blue bubbles, lower relative to baseline; red bubbles, higher relative to baseline. Bubble size corresponds to degree of relative change.

hypothesized that BAIBA might represent a link between the BCAA surge and the shift to lipid metabolism. In the current study, subjects underwent indirect calorimetry at the conclusion of the fast (11), enabling the calculation of the respiratory quotient (RQ), which indicates the relative degree to which different fuel sources are utilized: the more complete the shift to lipid and ketone utilization, the lower the RQ. We found an inverse association between BAIBA and RQ (Figure 5C), suggesting that a higher BAIBA surge is associated with a more complete transition to lipid metabolism. Whether BAIBA is functioning as a mediator of this shift in fuel utilization or is simply a marker of this shift cannot be determined by this analysis.

Metabolite cluster analysis reveals multiple discrete starvation phases. Having considered individual classes of metabolites, we next considered the possibility that an unbiased reassessment of the global data set would demonstrate previously unappreciated temporal metabolite patterns. Using the entire metabolite data set as input, inclusive of the prebaseline, baseline, starvation, and refeeding time points, we identified 7 statistically unique clusters (Figure 6A and Supplemental Table 5). Four clusters demonstrated a temporal pattern of starvation-mediated decline (Figure 6A, clusters 4-7). Clusters 4 and 7 demonstrated continual decline throughout the fast, with cluster 7 being distinguished by a rapid return to baseline with refeeding. Cluster 5 was relatively stable for the first 3 days of fasting and then declined through fasting days 9-10, followed by a further decline with refeeding. Cluster 6 declined to a nadir at day 3 before trending back to baseline. Clusters that displayed a pattern of decline during fasting were enriched with lipids and lipid metabolites, especially those with fewer double bonds, consistent with data shown in Figures 3 and 4. Three clusters demonstrated a pattern of relative increase during fasting. Cluster 3, consisting of bile acids and unsaturated CE, increased progressively over the course of the fast. Clusters 1 and 2 peaked earlier, on fasting days 3 and 7, respectively.

Cluster 1 was of particular interest because its early increase during fasting days 1-2 coincided with the surge in ketones (Figure 6B). Moreover, cluster 1 was enriched in fatty acids, the ketone substrate, and in amino acids and related metabolites, including BCAA and BAIBA, which were predictors of ketosis and the NEFA surge in our prior analyses. Given the observed inverse association between relative BAIBA levels and RQ (Figure 5C), which is an indicator of the degree of shift to lipid/ketone utilization, we considered whether the RQ association might be a more generally prevalent feature of cluster 1 metabolites. Of the 77 total metabolites, BAIBA was 1 of 3 that demonstrated an inverse correlation: BAIBA $\left(\mathrm{R}^{2}=0.46, P=0.046\right), \mathrm{SM}(22: 0)\left(\mathrm{R}^{2}=0.46, P=0.044\right)$, and $\gamma$-aminobutyric acid (GABA) $\left(\mathrm{R}^{2}=0.58, P=0.018\right)$. Two of these three metabolites (BAIBA and GABA) have been previously suggested to promote fatty acid oxidation in different contexts (20-22). Collectively, these data raise the possibility that cluster 1 marks an intermediate phase of starvation, bridging the early reliance on glucose metabolism and the subsequent shift to utilization of lipids for fuel.

Starvation-induced hypoleptinemia as a driver of the adaptive starvation response. If the cluster analyses mark temporally distinct phases of starvation, then we reasoned that they could be leveraged to elucidate additional candidate hormonal drivers. Included in the metabolomics platform utilized in this study are a number of corticosteroids, including cortisol. Cortisol, which is known as a mediator of lipolysis in adipose tissue (23), increased on days 1-2 of fasting (Figure 7A). We also measured leptin, which has previously been shown to decline during early starvation in humans, although the dynamics during a prolonged fast were unknown $(24,25)$. We observed a reduction in leptin - approximately $50 \%$ within the first day - an effect that is too acute to be related to reduction in adipose mass (Figure 7B).

With temporal dynamics of corticosteroids, leptin, and a number of previously reported metabolically relevant hormones, including insulin, glucagon, adiponectin, T3, and FGF21, we tested for correlates to the clusters identified in Figure 6 (Supplemental Table 6). We also included NEFA and ketones in the analysis as positive controls, given that NEFA release and ketosis are indicators of adaptive starvation. Indeed, NEFA was significantly correlated with each of the clusters, whereas ketones correlated with all of the clusters except for cluster 3. Cortisol or related corticosteroids were significantly associated with all 7 clusters. Insulin demonstrated statistically significant associations with clusters 6 and 7; whereas glucagon was significantly 
A

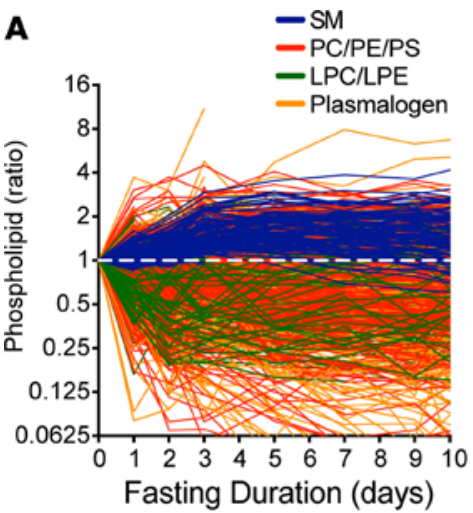

D

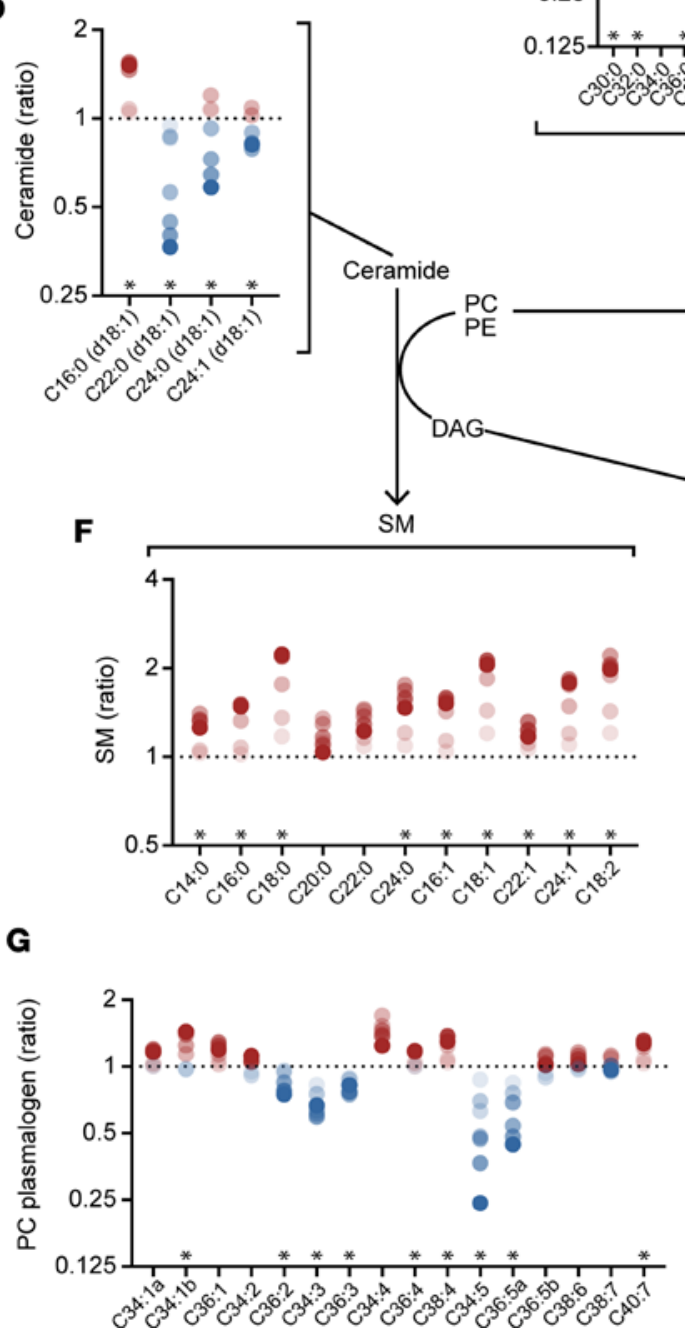

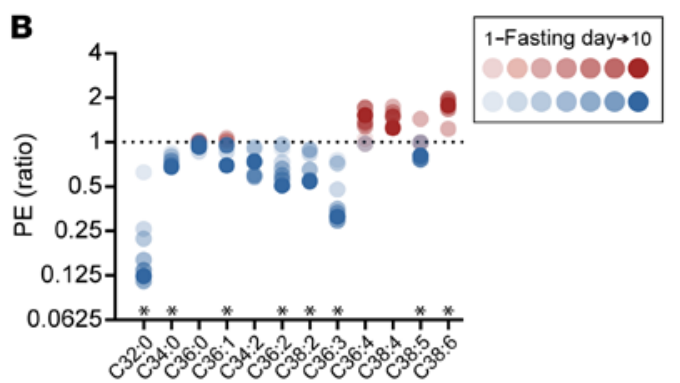
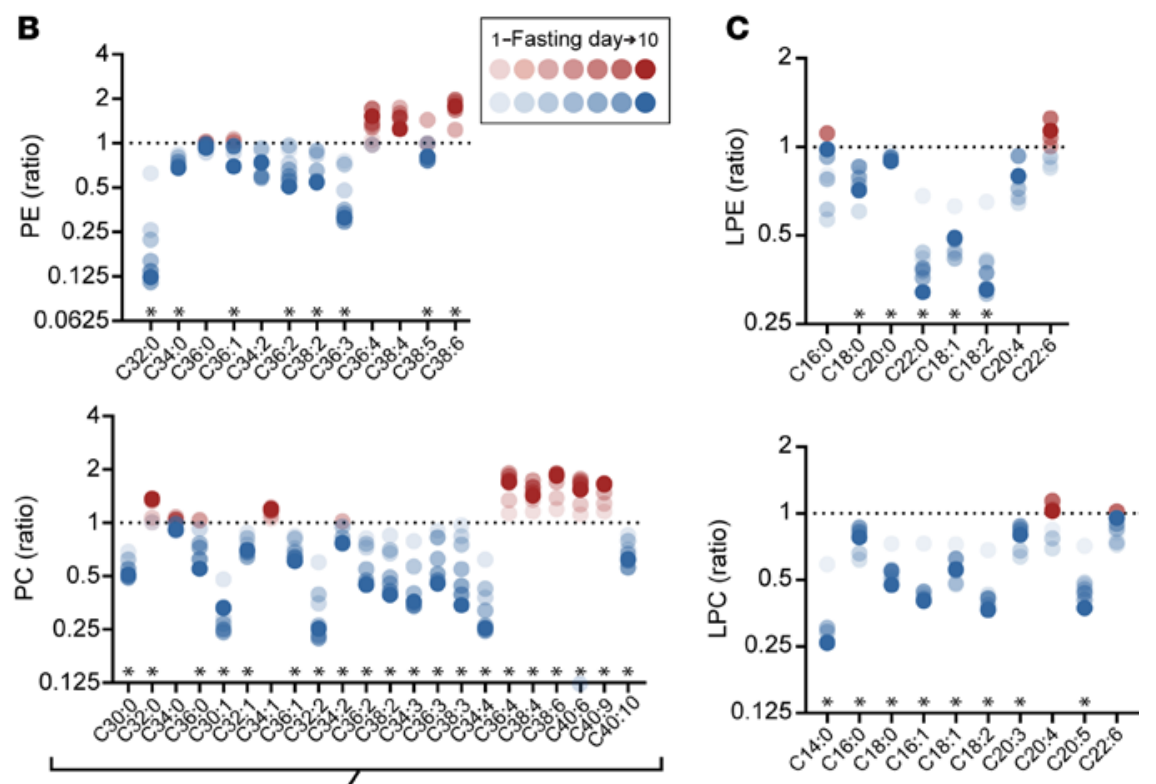

Figure 4. Starvation-mediated dynamics of plasma phospholipids. (A) Spaghetti plot of phospholipids for each subject, color-coded by phospholipid class. SM, sphingomyelin; PC, phosphatidylcholines; PE, phosphatidylethanolamines; PS, phosphatidylserines; LPC, lysophosphatidylcholines; LPE, lysophosphatidylethanolamines. (B) Mean phosphatidylethanolamine and phosphatidylcholine values relative to baseline (dashed line). (C) Mean lysophosphatidylethanolamine and lysophosphatidylcholine values relative to baseline (dashed line). Lysophosphatidylethanolamines/lysophosphatidylcholines are catabolic products resulting from fatty acid release from phosphatidylethanolamine and phosphatidylcholine, respectively (shown in B). (D) Mean ceramide levels relative to baseline (dashed line), which, together with PC and PE, serve as substrate for sphingomyelin synthesis. (E) Mean diacylglycerol (DAG) levels relative to baseline. Diacylglycerol is a byproduct of sphingomyelin synthesis. (F) Mean sphingomyelin levels relative to baseline (dashed line). (G) Mean phosphatidylcholine and phosphatidylethanolamine plasmalogen values relative to baseline (dashed line). (B-C) Blue dots signify negative levels relative to baseline; red dots signify positive levels relative to baseline. Dots become progressively darker as a function of fasting duration. Phospholipids listed on the $x$ axis are ordered with an increasing number of double bonds from left to right. ${ }^{*} P<0.05$, comparing day 10 value to baseline (false discovery rate adjustment for multiple comparisons). 
A
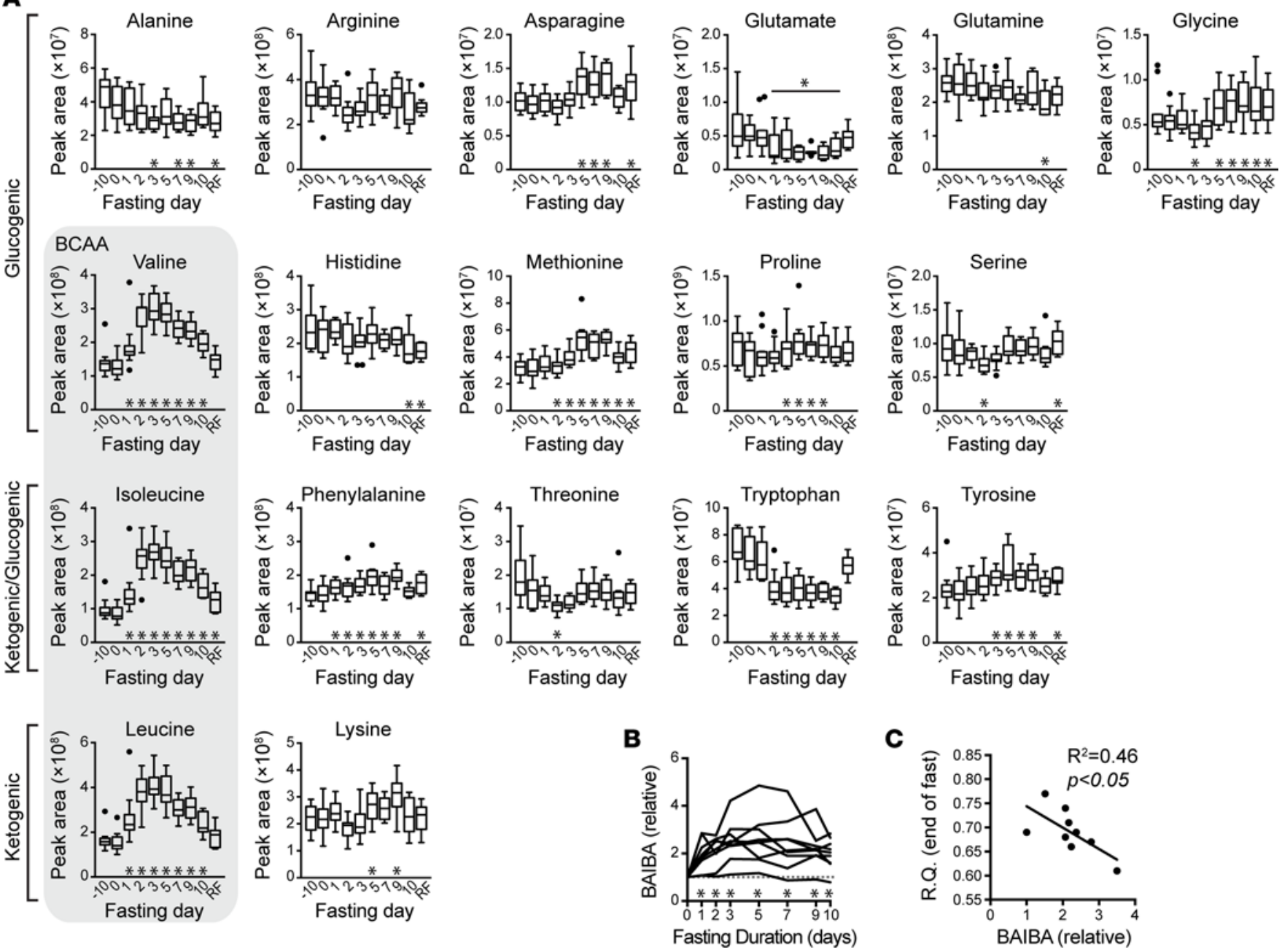

Figure 5. Starvation-mediated amino acid dynamics. (A) Tukey box plots of amino acids, grouped by whether they are glucogenic, both glucogenic and ketogenic, or ketogenic. The branched-chain amino acids (BCAA) are shown in the shaded box. ${ }^{*}$ Fasting and refed (RF) time points with adjusted $P<0.05$ relative to baseline (day 0). (B) Spaghetti plot for each subject of $\beta$-aminoisobutyric acid (BAIBA) levels relative to baseline (day 0 , dashed line). ${ }^{*}$ Time points with $P<0.05$ (false discovery rate adjustment for multiple comparisons) relative to baseline (day 0 ). (C) Univariate regression of respiratory quotient (R.Q.) on final fast day versus average relative BAIBA levels over the course of the fast.

associated with cluster 7. Interestingly, the early peaking cluster 1 was positively correlated with cortisol, cortexolone, ketones, and NEFA but inversely correlated with leptin. Fasting-induced hypoleptinemia in rodents has recently been implicated in a regulatory loop involving the HPA axis and increased corticosterone production, which promotes the adaptive shift from carbohydrate to lipid metabolism (9). If the rodent paradigm of a hypoleptinemia/HPA circuit is active in humans, we reasoned that the decline in leptin might correlate with the surge in cortisol (Figure 7C). Indeed, in a mixed-effects cubic model, we found an inverse correlation between the two hormones $(\mathrm{R}=-0.84 ; P<0.02$ ). Collectively, the acute decline in leptin (approximately $50 \%$ at day 1) coupled with its inverse correlation with cortisol and the early surging metabolite cluster 1 suggest a possible role for hypoleptinemia in the early adaptation to starvation in humans.

\section{Discussion}

Much of what is known about the adaptation to starvation in humans can be traced to classical studies conducted decades ago (3), in which individual metabolites or classes of metabolites were biochemically assayed in blood samples collected from fasting subjects. Such studies were typically conducted in obese individuals, rather than the normal to slightly overweight subjects studied here $(10,26)$. These studies, documenting the dynamics of circulating amino acids, were often inclusive of additional sampling across capillary beds and measurement of nitrogen content in urine, and led to the current paradigm of how humans 
A

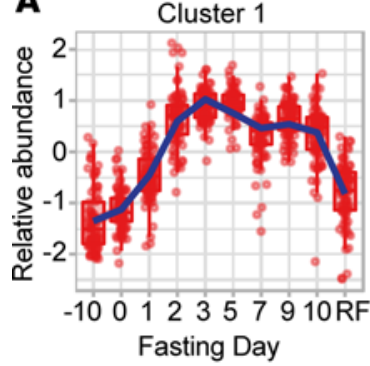

Fatty acid
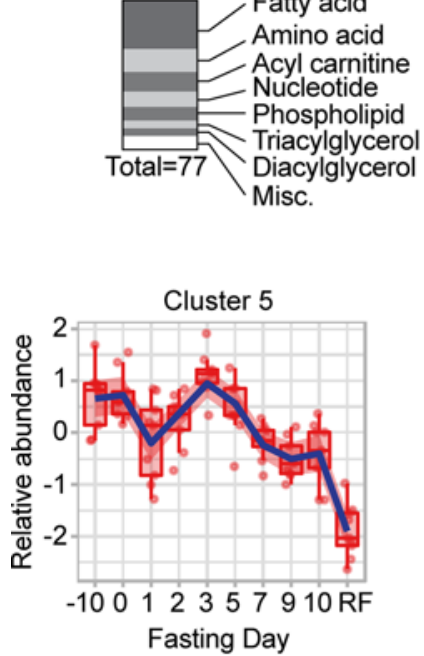

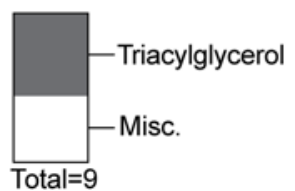

Cluster 2
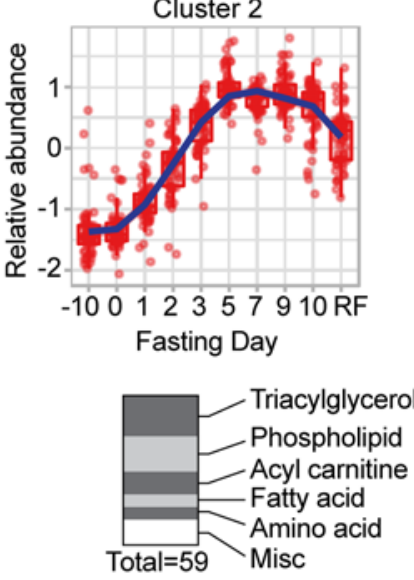

Cluster 6
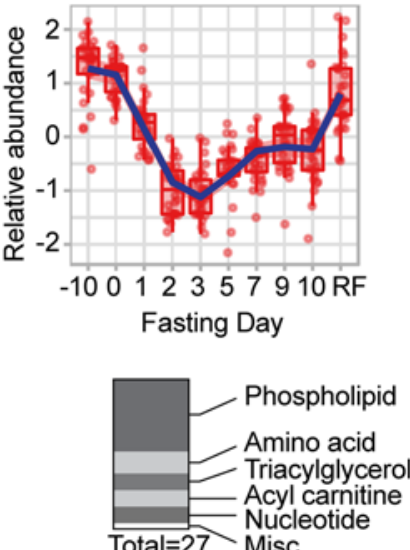

Cluster 3
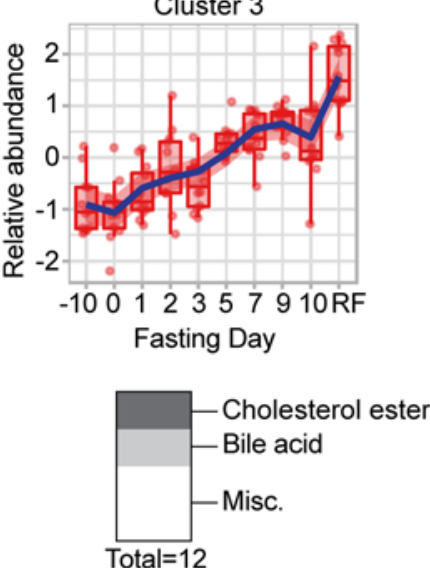

Cluster 7
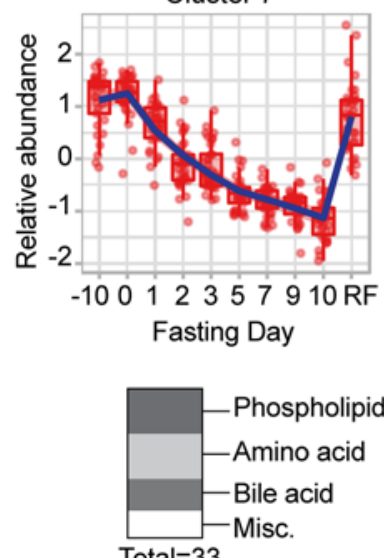
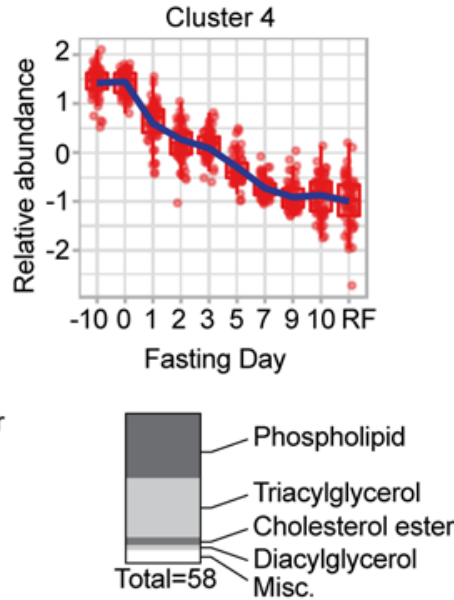

B

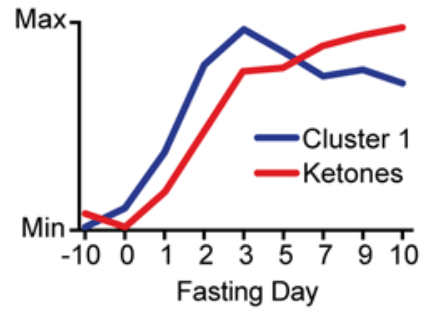

Figure 6. Temporal metabolite starvation phases revealed by cluster analysis. (A) Seven metabolite clusters were identified with discrete temporal patterns Below each cluster the number and type of metabolites contained in the cluster are listed (Supplemental Table 5 ). (B) Graph showing that cluster 1 (blue) and serum ketones (red) approximately coincide. Day -10, 10 days prior to initiation of fast; Day 0, baseline day; Day 10 , final fast day; RF, refeeding day 1.

survive prolonged starvation through protein sparing and by a shift from glucose to lipid catabolism. In this study, we profiled plasma metabolites in healthy subjects, who underwent a supervised, 0-calorie fast for 10 days. Plasma was serially sampled every 1-2 days, which, when coupled with the breadth of metabolites and other hormonal and clinical covariates, provides the most comprehensive longitudinal examination of circulating metabolites and potential regulating factors during human starvation. Although this study included a relatively small number of subjects, the metabolite shifts were quite uniform as disparate metabolite profiles converged, consistent with the activation of common adaptive mechanisms.

We first examined the data through a lens, similar to that through which plasma metabolites have historically been viewed: that is, we considered individual metabolites as part of predefined classes (Figures 2-5) and identified classical milestones in the starvation response, such as dynamic amino acid changes (3) and a uniform surge in NEFA (27), the identification of which reinforces the robust nature of the data set, while also providing temporal reference points for novel metabolites. We describe observations with this approach, facilitated by the frequency of sampling and by the fact that some classes, such as individual lipid species, have not been previously studied with the degree of granularity afforded by modern mass spectroscopic methods. The most unexpected theme was the modifying effect of carbon content and hydrogen saturation on lipid dynamics during starvation, indicated by the preferential surge in fatty acids, TAG, and CE with high-carbon and high double bond content. The fatty acid effect may be explainable, in part, by prior data in cultured adipocytes, demonstrating that lipolytic stimuli result in more rapid release of unsaturated fatty acids relative to fully 

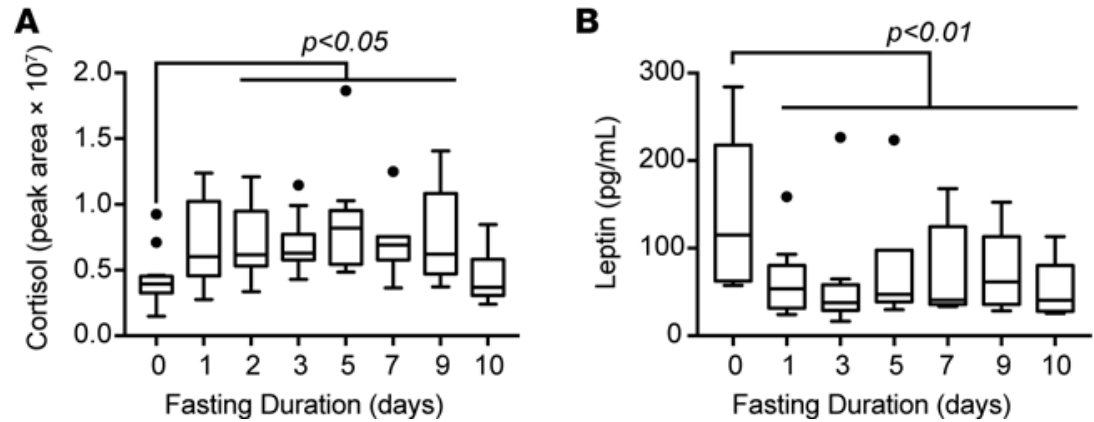

C

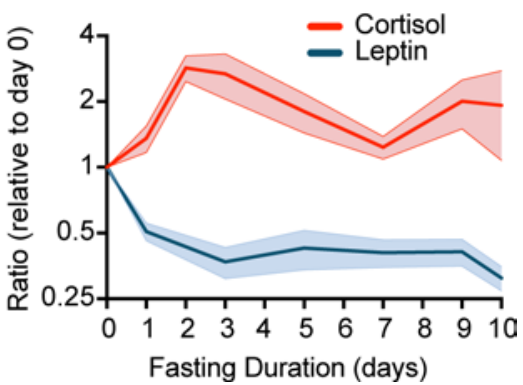

Figure 7. Acute starvation-mediated hypoleptinemia is associated with cortisol surge. (A) Relative cortisol levels during fasting measured using metabolomics platform. Data are expressed as Tukey box plots. (B) Leptin levels during fasting. Data are expressed as Tukey box plots. (C) Cortisol and leptin levels expressed as mean \pm SEM relative to baseline (day 0 ). Significance assigned for $P<0.05$ (false discovery rate adjustment for multiple comparisons) relative to baseline (day 0 ).

saturated species (28). In addition, we observed a decline in many plasmalogens, which generally have unsaturated acyl groups. If this decline is indicative of plasmalogen catabolism, then release of plasmalogen acyl groups could be an additional factor in the preferential surge in unsaturated fatty acids.

Mobilization of lipid stores requires the lipolytic release of NEFA because intact neutral lipids cannot cross the plasma membrane, and, therefore, the mechanism driving plasma levels of TAG with high-carbon and high double bond content is not easily reconciled based on mobilization of lipid stores alone. The two major sources of circulating TAG are gastrointestinal repackaging of dietary TAG into chylomicrons and de novo synthesis of TAG, which are packaged into VLDL by the liver. In the absence of a dietary source during starvation, the liver is the most likely source of new TAG. Indeed, prior studies have demonstrated a counterintuitive increase in TAG synthesis by the liver and development of frank liver steatosis during starvation $(9,29-31)$. It may be that mobilization of NEFA from adipose tissue exceeds immediate catabolic needs, and, therefore, the liver captures excess fatty acids for de novo TAG synthesis, some of which are retained in the liver and some of which are released into the circulation via VLDL. The surge in high-carbon and high double bond content TAG therefore may reflect the higher availability of mono- and PUFA as substrate for de novo TAG synthesis in the liver.

The directionally discordant amino acid dynamics illustrate a more general theme, that the net change in plasma metabolite concentrations is dictated by the complex interplay of mechanisms involved in substrate supply (e.g., amino acid release from intracellular compartments) and substrate utilization (e.g., uptake by the liver). Prior studies have demonstrated amino acid release from tissues, including skeletal muscle, during the first few days of human starvation (16). Despite this amino acid surge, some amino acids, such as alanine and tryptophan, decline during fasting. In the case of alanine, it has been demonstrated using invasive arterial and venous sampling that the net decline is attributable to high uptake by the liver for utilization as gluconeogenic substrate (10). This highlights an important limitation of our study: we did not perform invasive sampling of multiple vascular compartments. In some instances, however, the analysis of downstream metabolites provides additional clarity or presents intriguing new hypotheses. In the case of tryptophan, its decline with fasting occurred concomitant to an increase in some tryptophan-dependent metabolites, including metabolites in the kynurenine pathway and a trend toward increased serotonin. Modulation of tryptophan-dependent pathways involving serotonin signaling and kynurenic acid in lower organisms has been linked to regulation of feeding behavior (17-19). Therefore, the fact that an essential amino acid, such as tryptophan, may be preferentially shunted down these pathways raises the question of whether one or more of the associated metabolites may have an important role in attenuating the sensation of hunger during the adaptation to long-term starvation.

The surge in BCAA represented an additional striking amino acid divergence from baseline, confirming an effect observed in classic studies (3). Despite the fact that BCAA have divergent catabolic fates - valine is glucogenic, leucine is ketogenic, and isoleucine is both - they surged uniformly in circulation, suggesting that plasma levels of BCAA during starvation are regulated by common biochemical mechanisms. One possible explanation for the BCAA surge is decreased activity of a common catabolic enzyme. There is a shared proximal catabolic step in BCAA metabolism, driven by the BCAT enzyme. In mice, BCAT genetic loss of function results in high levels of BCAA (32). However, we found that several downstream BCAA 
catabolites also surged within 1-2 day of onset of fasting (Figure 5B and Supplemental Figure 4), suggesting that the BCAA surge is likely secondary to increased release into circulation, rather than a downstream block in substrate utilization. Our study was not designed to perform the requisite tracer studies and tissue analyses to address the mechanism of BCAA release. Even when such studies have been conducted, multiple mechanisms have been suggested, including increased release of intracellular free amino acid stores (33), increased protein catabolism (34), and decreased utilization of amino acids for protein synthesis (35). Such mechanisms could account for a more general flux of amino acids into circulation. Because movement of intracellular free amino acids across cell membranes requires the function of transporters that are specific for one or more amino acids, we speculate that functional augmentation of transporter(s) specific to BCAA at the plasma membrane may account for their particularly pronounced increase (36).

The diverging dynamics of individual metabolites within larger classes, as underscored by TAG (Figure 3) and amino acids (Figure 5), provided a rationale to reexamine the data, independent of metabolite class, but rather defined simply by directionality and timing of change. Unbiased cluster analysis revealed 7 clusters. Despite differences in directionality, timing of change, and representation from diverse metabolite classes, 6 of 7 clusters correlated with serum ketones and 7 of 7 clusters correlated with global NEFA (Supplemental Table 6). On the one hand, this reinforces the concept that the shift to lipid metabolism - as marked by the NEFA and ketone surge - is central to the adaptive response. Another view is that they may represent unique temporal phases of starvation. One cluster in particular (Figure 6A, cluster 1) demonstrated a pattern of early rise similar to the ketone surge, raising the question of whether it can be viewed as a biomarker of the switch from carbohydrate to lipid metabolism. In addition to individual fatty acids, which are substrate for ketone production, cluster 1 also included potentially bioactive metabolites, such as BCAA and related catabolites, which were also each individually associated with ketones and global NEFA. The potential physiological significance of the BCAA signal is supported by prior epidemiological studies demonstrating that plasma BCAA are biomarkers for disease states characterized by systemic metabolic changes, including cancer, obesity, and diabetes mellitus (37-39). Interestingly, mice genetically deficient in BCAT, which catalyzes the first step in BCAA catabolism, demonstrate markedly elevated BCAA but impaired ketone formation with fasting (32). This suggests that it may not be BCAA themselves, but a downstream BCAT-dependent BCAA catabolite, which facilitate ketogenesis. The valine catabolite, BAIBA, may represent one such bioactive mediator. Like BCAA, BAIBA is a member of the early surging cluster 1 . Moreover, the fasting-mediated increase in BAIBA is inversely correlated with RQ, a marker of the efficiency of the transition to lipid metabolism. BAIBA has also been shown in other contexts to augment fatty acid oxidation and ketogenesis $(20,21)$. Therefore, we speculate that cluster 1 is not only a biomarker for the transition from carbohydrate to lipid metabolism, but may also contain effectors, such as BAIBA, that contribute to the transition from carbohydrate to lipid metabolism.

Even if individual metabolites may function as bioactive effectors, such pathways are often tightly orchestrated by hormonal shifts. For example, the fasting-mediated decline in insulin has been attributed a central role in adaptive starvation (6-8), in part by driving the shift from lipid storage to lipolytic release of fatty acids from adipocytes $(3,40)$. We tested for associations between a panel of metabolically relevant hormones and the clusters, which we propose as markers of specific phases of starvation. In addition to corticosteroids, which are known to promote lipolysis, we found an association between several of the clusters and leptin, including an inverse correlation with early surging cluster 1 . Consistent with prior studies in humans and rodents, we observed a sharp decline in leptin levels within the first day of fasting $(24,25,41)$. Although leptin is produced by adipocytes and may correlate with adiposity, the pattern of acute change cannot be explained by the starvation-mediated contraction of adipose tissue mass. Leptin has been extensively studied as a regulator of energy homeostasis; however, the administration of leptin to obese, leptin-replete humans has at most modest effects on weight loss and energy homeostasis (42). Our data, therefore, support the emerging concept that it is the absence of leptin - or hypoleptinemia - that is of physiological relevance (43). This was presaged by a recent study in rats demonstrating a role for fasting-mediated hypoleptinemia as a causal driver of the shift to lipid metabolism in a mechanism involving the HPA axis and corticosterone stimulation of adipocyte lipolysis (9). Although multiple studies in rodents have shown that manipulation of leptin levels can effect the HPA axis and specifically cortisol levels, the degree to which the HPA axis is operative in metabolic control is controversial, particularly as it relates to glucose metabolism in diabetic states (44-46). Our current study design cannot address causality in the same manner as the rodent studies (9); however, our data suggest the possibility that hypoleptinemic stimulation of the HPA axis may also play a role in the transition from carbohydrate to lipid metabolism in human starvation. 
Identifying components of the starvation response that might effect long-term outcomes, such as cardiometabolic disease, is limited by our study design, which lacks outcome data or sampling beyond the initial refeeding day. However, the majority of the metabolites that change substantially during starvation rapidly approach baseline values within the first refeeding day. A notable exception was evident in subsets of TAG and CE, in which the shift favoring species with high-carbon and high double bond content was sustained after refeeding. A similar shift has been observed with acute exercise (15). Moreover, longitudinal epidemiological data predict that such a shift, if sustained, would be associated with a decreased risk of diabetes (15). Therefore, these data raise the intriguing hypothesis that periods of starvation may reprogram lipid metabolism in a beneficial way.

In summary, we present the longitudinal evolution of plasma metabolites during prolonged starvation in humans. Our study design, in which human volunteers were subjected to a well-defined, medically supervised inpatient fast, enables us to make causal connections between our intervention (fasting) and resultant metabolite shifts (Figures 1-4 and Figure 5A). Our additional secondary analyses, in which candidate metabolites were tested for associations with other metabolites, hormones, or functional covariates provide supportive evidence for specific hypotheses (Figure 5, B and C, and Figures 6 and 7), which will require prospective interventional studies to test for causality. Our data support a working model whereby the shift to lipid metabolism is marked by a cluster of metabolites from disparate classes that surges 1-2 days after fasting onset (Figure 6, cluster 1). This phase coincides with the onset of ketosis and is associated with candidate metabolites, such as BAIBA, with bioactive properties that may directly facilitate adaptive metabolic responses by promoting fatty acid oxidation and ketogenesis. This phase is also characterized by a state of acute hypoleptinemia and associated hypercortisolemia, a hormonal loop that we speculate may help orchestrate the shift from carbohydrate to lipid metabolism. These data undoubtedly capture additional biologically relevant events beyond those discussed in this manuscript, and, therefore, we expect that these data will be a resource for investigations of physiological and pathophysiological states influenced by the metabolism of fasting and feeding.

\section{Methods}

\section{Study protocol}

As previously described (11), we studied 12 healthy subjects ( 8 women and 4 men; mean age, 30.7 years; age range, 21.8-48.3 years), with BMI in the normal or overweight range (BMI range, 22.7-29.3 kg/ $\mathrm{m}^{2}$ ). We recruited the subjects through online advertisements. All subjects had normal thyroid function and regular menstrual cycles (women). We excluded anyone with a history of an eating disorder or chronic illness. At a prebaseline visit, blood was drawn after an overnight ( $\geq 8 \mathrm{~h}$ ) fast. Approximately 10 days later, after an overnight fast, subjects were admitted to the Center for Clinical Investigation at Brigham and Women's Hospital for the 0 -calorie fasting protocol. Subjects were allowed water ad libitum and daily received a multivitamin, 20 meq potassium chloride, and $200 \mathrm{mg}$ allopurinol, as previously described (11). Resting energy expenditure was measured at baseline and at fasting conclusion, using indirect calorimetry (VMAX Encore 29 metabolic cart, Carefusion). Blood samples were drawn at the prebaseline visit; baseline (day 0); days 1, $2,3,5,7,9$, and 10; and approximately 24 hours after refeeding.

\section{Metabolomics analysis}

Plasma metabolites were measured using liquid chromatography tandem mass spectrometry (LC-MS) as previously described (47-49). MS peaks were integrated using TraceFinder 3.3 (Thermo Fisher Scientific) and Progenesis QI (Nonlinear Dynamics). Metabolite identities were determined based on comparison to reference standards and reference samples.

Lipid method. Plasma lipids were profiled using a Shimadzu Nexera X2 U-HPLC (Shimadzu Corp.). Lipids were extracted from plasma $(10 \mu \mathrm{l})$ using $190 \mu \mathrm{l}$ isopropanol containing 1,2-didodecanoyl-sn-glycero-3-phosphocholine (Avanti Polar Lipids). After centrifugation, supernatants were injected directly onto a $100 \mathrm{~mm} \times 2.1 \mathrm{~mm}, 1.7 \mu \mathrm{m}$ ACQUITY BEH C8 column (Waters). The column was eluted isocratically with $80 \%$ mobile phase A (95:5:0.1 vol/vol/vol, $10 \mathrm{mM}$ ammonium acetate/methanol/formic acid) for 1 minute followed by a linear gradient to $80 \%$ mobile phase B (99.9:0.1 vol/vol methanol/ formic acid) over 2 minutes, a linear gradient to $100 \%$ mobile phase B over 7 minutes, and then 3 minutes at $100 \%$ mobile phase B. MS analyses were carried out using electrospray ionization (ESI) in the positive ion mode using full-scan analysis over $200-1,100 \mathrm{~m} / z$ at 70,000 resolution and $3-\mathrm{Hz}$ data acquisition 
rate. Other MS settings were as follows: sheath gas, 50; in source CID, $5 \mathrm{eV}$; sweep gas, 5; spray voltage, $3 \mathrm{kV}$; capillary temperature, $300^{\circ} \mathrm{C}$; heater temperature, $300^{\circ} \mathrm{C}$; and S-lens $\mathrm{RF}, 60$. Lipid identities were denoted by total acyl carbon number and total number of double bonds.

Intermediate polarity method. Metabolites of intermediate polarity, including free fatty acids and bile acids, were profiled using a Shimadzu Nexera X2 U-HPLC (Shimadzu Corp.) coupled to a Q Exactive (Thermo Fisher Scientific). Plasma samples $(30 \mu \mathrm{l})$ were extracted using $90 \mu \mathrm{l}$ methanol containing PGE2-d4 as an internal standard (Cayman Chemical Co.) and centrifuged $\left(10 \mathrm{~min}, 9,000 \mathrm{~g}, 4^{\circ} \mathrm{C}\right)$. The supernatants $(10 \mu \mathrm{l})$ were injected onto a $150 \mathrm{~mm} \times 2.1 \mathrm{~mm}$ ACQUITY BEH C18 column (Waters). The column was eluted isocratically at a flow rate of $450 \mu 1 / \mathrm{min}$ with $20 \%$ mobile phase A ( $0.01 \%$ formic acid in water) for 3 minutes followed by a linear gradient to $100 \%$ mobile phase B $(0.01 \%$ acetic acid in acetonitrile) over 12 minutes. Negative ion mode MS analyses used ESI and full-scan analysis over 70-850 m/z at 70,000 resolution and 3-Hz data acquisition rate. Additional MS settings were as follows: ion spray voltage, $-3.5 \mathrm{kV}$; capillary temperature, $320^{\circ} \mathrm{C}$; probe heater temperature, $300^{\circ} \mathrm{C}$; sheath gas, 45 ; auxiliary gas, 10 ; and S-lens RF level 60.

Positively charged polar metabolites. Polar metabolites were profiled using a Shimadzu Nexera X2 U-HPLC (Shimadzu Corp.) coupled to a Q Exactive (Thermo Fisher Scientific). Metabolites were extracted from plasma (10 $\mu \mathrm{l})$ using $90 \mu \mathrm{l}$ of 74.9:24.9:0.2 (vol/vol/vol) acetonitrile/methanol/formic acid containing stable isotope-labeled internal standards (valine-d8, MilliporeSigma, and phenylalanine-d8, Cambridge Isotope Laboratories). The samples were centrifuged $\left(10 \mathrm{~min}, 9,000 \mathrm{~g}, 4^{\circ} \mathrm{C}\right)$, and the supernatants were injected directly onto a $150 \mathrm{~mm} \times 2 \mathrm{~mm}, 3 \mu \mathrm{m}$ Atlantis HILIC column (Waters). The column was eluted isocratically at a flow rate of $250 \mu \mathrm{l} / \mathrm{min}$ with $5 \%$ mobile phase $\mathrm{A}$ ( $10 \mathrm{mM}$ ammonium formate and $0.1 \%$ formic acid in water) for 0.5 minute followed by a linear gradient to $40 \%$ mobile phase B (acetonitrile with $0.1 \%$ formic acid) over 10 minutes. Positive ion mode MS data were acquired using ESI and full-scan analysis over 70-800 $\mathrm{m} / \mathrm{z}$ at 70,000 resolution and $3-\mathrm{Hz}$ data acquisition rate. Other $\mathrm{MS}$ settings were as follows: sheath gas, 40; sweep gas, 2; spray voltage, $3.5 \mathrm{kV}$; capillary temperature, $350^{\circ} \mathrm{C}$; heater temperature, $300^{\circ} \mathrm{C}$; and S-lens $\mathrm{RF}, 40$.

Negatively charged polar metabolites. Polar metabolites were profiled using a Shimadzu Nexera X2 U-HPLC (Shimadzu Corp.) coupled to a Q Exactive Plus (Thermo Fisher Scientific). Metabolites were extracted from plasma (30 $\mu \mathrm{l}$ ) using $120 \mu \mathrm{l}$ of $80 \%$ methanol containing inosine-15N4, thymine-d4, and glycocholate-d4 internal standards (Cambridge Isotope Laboratories). The samples were centrifuged (10 min, $9,000 \mathrm{~g}, 4^{\circ} \mathrm{C}$ ), and the supernatants were injected directly onto a $150 \mathrm{~mm} \times 2.0 \mathrm{~mm}$ Luna NH2 column (Phenomenex). The column was eluted at a flow rate of $400 \mu 1 / \mathrm{min}$, with initial conditions of $10 \%$ mobile phase A (20 mM ammonium acetate and $20 \mathrm{mM}$ ammonium hydroxide in water) and $90 \%$ mobile phase B (10 mM ammonium hydroxide in 75:25 vol/vol acetonitrile/methanol) followed by a 10-minute linear gradient to $100 \%$ mobile phase A. Negative ion mode MS data were acquired using ESI and full-scan analysis over $70-750 \mathrm{~m} / z$ at 70,000 resolution and 3-Hz data acquisition rate. Additional MS settings were as follows: ion spray voltage, $-3.0 \mathrm{kV}$; capillary temperature, $350^{\circ} \mathrm{C}$; probe heater temperature, $325^{\circ} \mathrm{C}$; sheath gas, 55; auxiliary gas, 10; and S-lens RF, level 50.

\section{Biochemical assessment}

$\beta$-Hydroxybutyrate (ketones), insulin, glucagon, FGF21, adiponectin, T3, glucose, and baseline lipids (including a calculated LDL) were measured as previously reported (11). Leptin was measured by solid-phase sandwich ELISA (R\&D Systems), with an intra-assay CV of 3\%-3.3\% and an inter-assay CV of $3.5 \%-5.4 \%$.

\section{Statistics}

We performed a PCA analysis using the DEGreport BioC package and then utilized a Kendall rank test to assess for correlations between the principal components and clinical covariates. To assess for changes in metabolites during fasting, we performed differential expression using the limma BioC package (50) comparing each fasting time point to baseline (day 0 ) and correcting by subject. We report $P$ values adjusted for the multiple comparisons to baseline using the false discovery rate method. For the metabolite cluster analysis, we calculated all pairwise correlations between metabolites and then performed a divisive hierarchical clustering with the cluster Bioc package, using a minimum cluster size of 6 metabolites. To assess the association between changes in the metabolites and NEFA and ketones, we performed a differential expression analysis with limma (50), correcting for subject and time. To assess the correlations between the 7 clusters and the 13 hormonal variables, we performed a correlation test analysis and corrected by the false discovery rate to control for multitesting error. We used a mixed-effects cubic model to assess the relation- 
ship between longitudinal measurements of leptin and cortisol. Code for analyses is available at https://

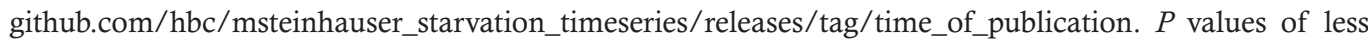
than 0.05 were considered significant.

\section{Study approval}

This study was approved by the Partners HealthCare Institutional Review Board (Boston, Massachusetts, USA) and complied with the Health Insurance Portability and Accountability Act guidelines. Written informed consent was obtained from all subjects.

\section{Author contributions}

MLS designed and conducted the study, acquired and analyzed data, and wrote the manuscript; BAO acquired and analyzed data; JO acquired data; ML acquired data; KAP acquired and analyzed data; HL designed the study and analyzed data; LP analyzed data; AK designed the study; GIS critically analyzed data; CBC designed the study and acquired and critically analyzed data; and PKF designed and conducted the study, acquired and analyzed data, and wrote the manuscript. All authors critically reviewed and approved the manuscript.

\section{Acknowledgments}

This work was supported by the KL2/Catalyst Medical Research Investigator Training award from the Harvard Catalyst/Harvard Clinical and Translational Science Center (National Center for Advancing Translational Sciences, NIH award KL2 TR002542), NIH award UL 1TR002541, and the Claflin Distinguished Scholar Award, Massachusetts General Hospital. The content is solely the responsibility of the authors and does not necessarily represent the official views of Harvard Catalyst, Harvard University and its affiliated academic healthcare centers, or the NIH.

Address correspondence to: Matthew L. Steinhauser, Brigham and Women's Hospital, 77 Avenue Louis Pasteur, 4th Floor, Boston, Massachusetts 02115, USA. Phone: 617.525.4716; Email: msteinhauser@bwh. harvard.edu. Or to: Pouneh K. Fazeli, Massachusetts General Hospital, 55 Fruit Street, Bulfinch 457, Boston, Massachusetts 02114, USA. Phone: 617.726.3870; Email: pkfazeli@partners.org.

1. Blossner M, de Onis M. Malnutrition: quantifying the health impact at national and local levels. WHO Environmental Burden of Disease Series. http://apps.who.int/iris/handle/10665/43120. Accessed August 2, 2018.

2. Heilbronn LK, Ravussin E. Calorie restriction and aging: review of the literature and implications for studies in humans. $A m J$ Clin Nutr. 2003;78(3):361-369.

3. Cahill GF. Starvation in man. N Engl J Med. 1970;282(12):668-675.

4. Rothman DL, Magnusson I, Katz LD, Shulman RG, Shulman GI. Quantitation of hepatic glycogenolysis and gluconeogenesis in fasting humans with 13C NMR. Science. 1991;254(5031):573-576.

5. Marliss EB, Aoki TT, Unger RH, Soeldner JS, Cahill GF. Glucagon levels and metabolic effects in fasting man. J Clin Invest. 1970;49(12):2256-2270.

6. Yoon JC, et al. Control of hepatic gluconeogenesis through the transcriptional coactivator PGC-1. Nature. 2001;413(6852):131-138

7. Herzig S, et al. CREB regulates hepatic gluconeogenesis through the coactivator PGC-1. Nature. 2001;413(6852):179-183.

8. Puigserver P, et al. Insulin-regulated hepatic gluconeogenesis through FOXO1-PGC-1alpha interaction. Nature. 2003;423(6939):550-555.

9. Perry RJ, et al. Leptin mediates a glucose-fatty acid cycle to maintain glucose homeostasis in starvation. Cell. 2018;172(12):234-248.e17.

10. Felig P, Owen OE, Wahren J, Cahill GF. Amino acid metabolism during prolonged starvation. J Clin Invest. 1969;48(3):584-594.

11. Fazeli PK, et al. FGF21 and the late adaptive response to starvation in humans. J Clin Invest. 2015;125(12):4601-4611.

12. Gordon RS, Cherkes A. Unesterified fatty acid in human blood plasma. J Clin Invest. 1956;35(2):206-212.

13. Hack A, et al. Monitoring of ketogenic diet for carnitine metabolites by subcutaneous microdialysis. Pediatr Res. 2006;60(1):93-96.

14. Stout RW, Henry RW, Buchanan KD. Triglyceride metabolism in acute starvation: the role of secretin and glucagon. Eur J Clin Invest. 1976;6(2):179-185.

15. Rhee EP, et al. Lipid profiling identifies a triacylglycerol signature of insulin resistance and improves diabetes prediction in humans. J Clin Invest. 2011;121(4):1402-1411.

16. Pozefsky T, Tancredi RG, Moxley RT, Dupre J, Tobin JD. Effects of brief starvation on muscle amino acid metabolism in nonobese man. J Clin Invest. 1976;57(2):444-449.

17. Sze JY, Victor M, Loer C, Shi Y, Ruvkun G. Food and metabolic signalling defects in a Caenorhabditis elegans serotonin-synthesis mutant. Nature. 2000;403(6769):560-564.

18. Curzon G. Serotonin and appetite. Ann N Y Acad Sci. 1990;600:521-530.

19. Lemieux GA, Cunningham KA, Lin L, Mayer F, Werb Z, Ashrafi K. Kynurenic acid is a nutritional cue that enables behavioral 
plasticity. Cell. 2015;160(1-2):119-131.

20. Maisonneuve C, et al. Effects of zidovudine, stavudine and beta-aminoisobutyric acid on lipid homeostasis in mice: possible role in human fat wasting. Antivir Ther (Lond). 2004;9(5):801-810.

21. Roberts LD, et al. $\beta$-Aminoisobutyric acid induces browning of white fat and hepatic $\beta$-oxidation and is inversely correlated with cardiometabolic risk factors. Cell Metab. 2014;19(1):96-108.

22. Sen S, et al. $\gamma$-Aminobutyric acid is synthesized and released by the endothelium: potential implications. Circ Res. 2016;119(5):621-634.

23. Djurhuus CB, et al. Effects of cortisol on lipolysis and regional interstitial glycerol levels in humans. Am J Physiol Endocrinol Metab. 2002;283(1):E172-E177.

24. Boden G, Chen X, Mozzoli M, Ryan I. Effect of fasting on serum leptin in normal human subjects. J Clin Endocrinol Metab. 1996;81(9):3419-3423.

25. Chan JL, Heist K, DePaoli AM, Veldhuis JD, Mantzoros CS. The role of falling leptin levels in the neuroendocrine and metabolic adaptation to short-term starvation in healthy men. J Clin Invest. 2003;111(9):1409-1421.

26. Felig P, Pozefsky T, Marliss E, Cahill GF. Alanine: key role in gluconeogenesis. Science. 1970;167(3920):1003-1004

27. Hanson PG, Johnson RE, Zaharko DS. Correlation between ketone body and free fatty acid concentrations in the plasma during early starvation in man. Metab Clin Exp. 1965;14(10):1037-1040.

28. Raclot T, Groscolas R. Differential mobilization of white adipose tissue fatty acids according to chain length, unsaturation, and positional isomerism. J Lipid Res. 1993;34(9):1515-1526.

29. Badaloo A, Reid M, Soares D, Forrester T, Jahoor F. Relation between liver fat content and the rate of VLDL apolipoprotein B-100 synthesis in children with protein-energy malnutrition. Am J Clin Nutr. 2005;81(5):1126-1132.

30. van Ginneken V, et al. Metabolomics (liver and blood profiling) in a mouse model in response to fasting: a study of hepatic steatosis. Biochim Biophys Acta. 2007;1771(10):1263-1270.

31. Browning JD, Baxter J, Satapati S, Burgess SC. The effect of short-term fasting on liver and skeletal muscle lipid, glucose, and energy metabolism in healthy women and men. J Lipid Res. 2012;53(3):577-586.

32. She P, et al. Disruption of BCATm in mice leads to increased energy expenditure associated with the activation of a futile protein turnover cycle. Cell Metab. 2007;6(3):181-194.

33. Giesecke K, Magnusson I, Ahlberg M, Hagenfeldt L, Wahren J. Protein and amino acid metabolism during early starvation as reflected by excretion of urea and methylhistidines. Metab Clin Exp. 1989;38(12):1196-1200.

34. Fryburg DA, Barrett EJ, Louard RJ, Gelfand RA. Effect of starvation on human muscle protein metabolism and its response to insulin. Am J Physiol. 1990;259(4 Pt 1):E477-E482.

35. Rennie MJ, Edwards RH, Halliday D, Matthews DE, Wolman SL, Millward DJ. Muscle protein synthesis measured by stable isotope techniques in man: the effects of feeding and fasting. Clin Sci. 1982;63(6):519-523.

36. Fukuhara D, et al. Protein characterization of NA+-independent system L amino acid transporter 3 in mice: a potential role in supply of branched-chain amino acids under nutrient starvation. Am J Pathol. 2007;170(3):888-898.

37. Wang TJ, et al. Metabolite profiles and the risk of developing diabetes. Nat Med. 2011;17(4):448-453.

38. Mayers JR, et al. Elevation of circulating branched-chain amino acids is an early event in human pancreatic adenocarcinoma development. Nat Med. 2014;20(10):1193-1198.

39. Newgard CB, et al. A branched-chain amino acid-related metabolic signature that differentiates obese and lean humans and contributes to insulin resistance. Cell Metab. 2009;9(4):311-326.

40. Butcher RW. Role of cyclic AMP in hormone actions. N Engl J Med. 1968;279(25):1378-1384.

41. Ahima RS, et al. Role of leptin in the neuroendocrine response to fasting. Nature. 1996;382(6588):250-252.

42. Heymsfield SB, et al. Recombinant leptin for weight loss in obese and lean adults: a randomized, controlled, dose-escalation trial. JAMA. 1999;282(16):1568-1575.

43. Ravussin Y, Leibel RL, Ferrante AW. A missing link in body weight homeostasis: the catabolic signal of the overfed state. Cell Metab. 2014;20(4):565-572.

44. Perry RJ, et al. Leptin reverses diabetes by suppression of the hypothalamic-pituitary-adrenal axis. Nat Med. 2014;20(7):759-763.

45. Morton GJ, Meek TH, Matsen ME, Schwartz MW. Evidence against hypothalamic-pituitary-adrenal axis suppression in the antidiabetic action of leptin. J Clin Invest. 2015;125(12):4587-4591.

46. Oberlin D, Buettner C. How does leptin restore euglycemia in insulin-deficient diabetes? J Clin Invest. 2017;127(2):450-453.

47. Mascanfroni ID, et al. Metabolic control of type 1 regulatory T cell differentiation by AHR and HIF1- $\alpha$. Nat Med. 2015;21(6):638-646.

48. O'Sullivan JF, et al. Dimethylguanidino valeric acid is a marker of liver fat and predicts diabetes. J Clin Invest. 2017;127(12):4394-4402.

49. Rowan $\mathrm{S}$, et al. Involvement of a gut-retina axis in protection against dietary glycemia-induced age-related macular degeneration. Proc Natl Acad Sci USA. 2017;114(22):E4472-E4481.

50. McCarthy DJ, Smyth GK. Testing significance relative to a fold-change threshold is a TREAT. Bioinformatics. 2009;25(6):765-771. 